\title{
Investigation of Non-Linearity in Dielectric Response Measurements for Transformer Insulation Diagnosis
}

\author{
Atefeh Dehghani Ashkezari, Student Member, IEEE, Hui Ma, Member, IEEE, Tapan K.Saha, Senior Member, \\ IEEE, and Chandima Ekanayake, Member, IEEE
}

\begin{abstract}
Dielectric response measurement is emerging as one of the potential non-destructive tools for transformer insulation diagnosis. Studies have been conducted to derive equivalent circuits for dielectric response of transformer oil/paper insulation system, which are normally based on a linear RC model. However, in practice transformer insulation systems may exhibit nonlinear polarisation behaviour. This paper investigates the nonlinearity of the dielectric response of transformer insulation under high excitation voltages. Polarisation and depolarisation current (PDC) measurement is performed on a distribution transformer under different voltages to study the nonlinearity of its dielectric response. Based on these measurements a nonlinear transformer insulation model is proposed, in which a current controlled voltage source (CCVS) is augmented to the wellknown Debye model to approximate the nonlinear effect.
\end{abstract}

Index Terms-Power transformer; oil-paper insulation; dielectric response; polarisation and depolarisation current measurement; equivalent circuit; modelling.

\section{INTRODUCTION}

$\mathrm{P}$ OWER transformer is one of the most critical and expensive assets in electricity supply chain. To achieve sustainable reliability of electricity networks, the condition based asset management plan needs to be developed for power transformers, which can provide a comprehensive assessment of transformer conditions and advise on maintenance and operation schedule to minimise total life cycle costs of transformers. Within the condition based asset management of power transformer, the priority attention should be directed to transformer insulation monitoring, diagnosis, and data interpretation.

A number of transformer insulation monitoring and diagnose techniques have been developed [1], [2]. Among them, time domain dielectric response method, i.e. the polarisation and depolarisation current (PDC) measurement technique has gained the popularity across the research community and utility recently [3].

In PDC measurements and the corresponding results interpretation, it is assumed transformer insulation system behaves linearly under different testing voltages. However, the study of this paper reveals that transformer insulation system may exhibit nonlinearity under different testing voltages. By investigating the correlations between PDC measurements and testing voltages, this paper proposes a nonlinear model for dielectric response of transformer insulation system, which augments a current controlled voltage source (CCVS) to the well-known Debye model.

The organization of this paper is as follows. Section II reviews the theory of dielectric response measurements. Section III discusses the nonlinearity in time domain dielectric response, i.e. PDC measurements. Section IV presents the results of laboratory experiments and discusses the correlation between polarisation currents and testing voltages. Section V proposed a nonlinear model, which incorporates a CCVS to the extended Debye model to represent the nonlinear characteristics on dielectric response of transformer insulation. Section VI concludes the paper.

\section{REVIEW OF DIELECTRIC RESPONSE MEASUREMENTS}

This section provides a brief review of the dielectric behaviour of insulation materials and the principles of polarisation and depolarisation current (PDC) techniques.

\section{A. Linear Dielectric response Equivalent Circuit}

A number of studies [4], [5] have proposed equivalent circuits to model the electrical response of oil/paper insulation systems. These equivalent circuits are normally the extensions of the Debye theory, which is based on a simple RC model [3]. The Debye theory states that in the presence of an electric field E, a polarisation current will develop as the dipoles align in the direction of the field [6]. When the dipoles are no longer subjected to E, they return back to their original states [6], [7].

In dielectric polymer, each polar group exhibits different configuration to its neighbouring molecules. Thus, each group may have a different response time when an electric field is supplied [6]. Fig.1 depicts response of different dipole groups under an external field [3], [4], [7], [8] and [9]. In Fig.1, $\mathrm{R}_{\mathrm{i}}-\mathrm{C}_{\mathrm{i}}$ represent the response of dipoles that are randomly distributed, and the associated time constants of these dipoles are given by $\tau_{\mathrm{i}}=\mathrm{R}_{\mathrm{i}} \mathrm{C}_{\mathrm{i}} . \quad \mathrm{C}_{0}$ is the geometric capacitance (measured capacitance at $50 \mathrm{~Hz}$ divided by relative permittivity) and $\mathrm{R}_{0}$ is insulation resistance. The above circuit represents the summation of various relaxation mechanisms that appear at different locations within the transformer insulation system.

Atefeh Dehghani Ashkezari (atefeh@uq.edu.au), Hui Ma (huima@itee.uq.edu.au), T.K. Saha (saha@itee.uq.edu.au) and Chandima Ekanayake (chandima@itee.uq.edu.au) are with the School of ITEE, The University of Queensland, St Lucia, Brisbane, QLD-4072, Australia. 


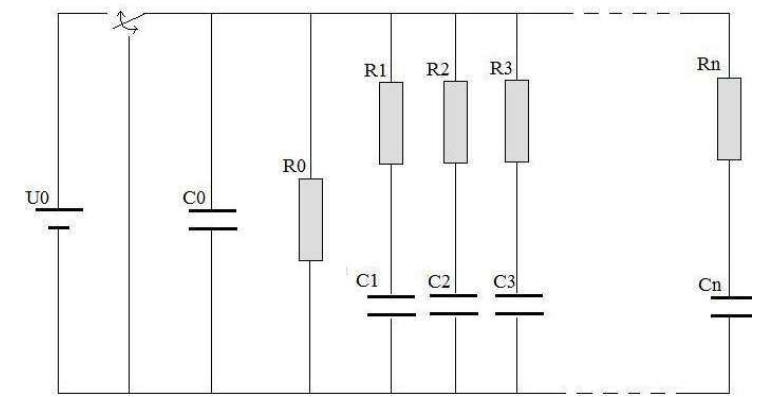

Fig. 1. Equivalent circuit to model a linear dielectric

\section{B. Polarisation and Depolarisation Current Measurement} (PDC)

In PDC measurements, a DC voltage of magnitude $\mathrm{U}_{0}$ is applied to a test object for a considerable long period [10]. During this charging period, the polarisation current following through this object can be expressed as:

$$
{ }_{p}^{i}(t)=C_{0} U_{0}\left[\frac{\sigma}{\varepsilon_{0}}+f(t)\right]
$$

Where $\sigma$ is DC conductivity, $\varepsilon_{0}$ is the permittivity of vacuum, $f(t)$ is the response function and $\mathrm{C}_{0}$ is the geometric capacitance (measured capacitance divided by relative permittivity) of the dielectric material [11].

The voltage source is then replaced with short circuit to leave the object discharge [10]. The resultant depolarisation current is expressed as:

$$
i_{d}(t)=-C_{0} U_{0}\left[f(t)-f\left(t+t_{p}\right)\right]
$$

The overall process is summarized in Fig. 2.

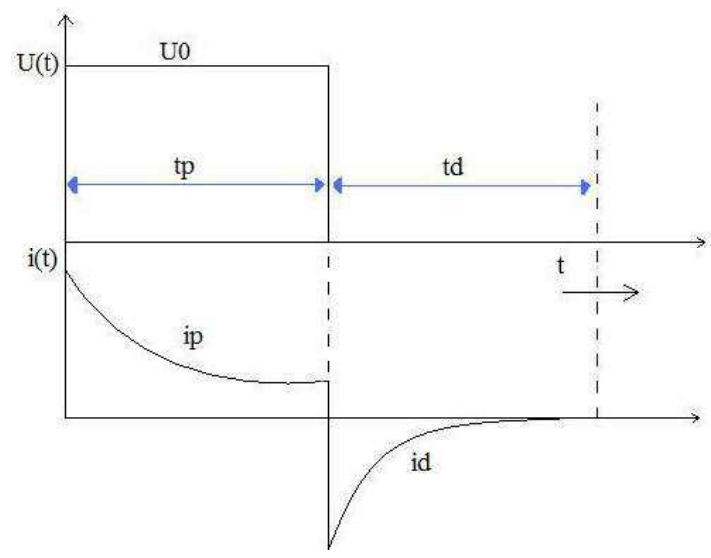

Fig. 2. Waveform of polarisation and depolarisation currents

\section{NONLINEARITY IN DIELECTRIC RESPONSE MEASUREMENTS}

In a nonlinear system, the difference between charging and discharging currents is equal to the time-independent direct current [12]:

$$
i_{c}(t)-i_{d}(t)=i_{0}(t)
$$

Fig. 3 shows an example of this property, which is obtained from the measured PDC currents on a stearic acid sample under various amplitudes of the applied voltages and temperatures [12]. At the lower temperature and voltage, the charging and discharging currents are quite similar in the short time range. In this condition since system is linear, Equation 4 is not applicable and time-independent direct current is equal to zero. However, if the temperature or amplitude of the voltage is increased sufficiently, the situation will be changed and a complete crossing over of the charging and discharging currents in the short time range will be occurred. Jonscher [12] suggested that the phenomenon of such crossing over is due to the injection of space charge during the charging process. This phenomenon leads to a gradual accumulation of excess charge density near one or both electrodes [12]. Therefore, the process of space charge injection is significantly non-linear and easily influenced by factors such as temperature and voltage.

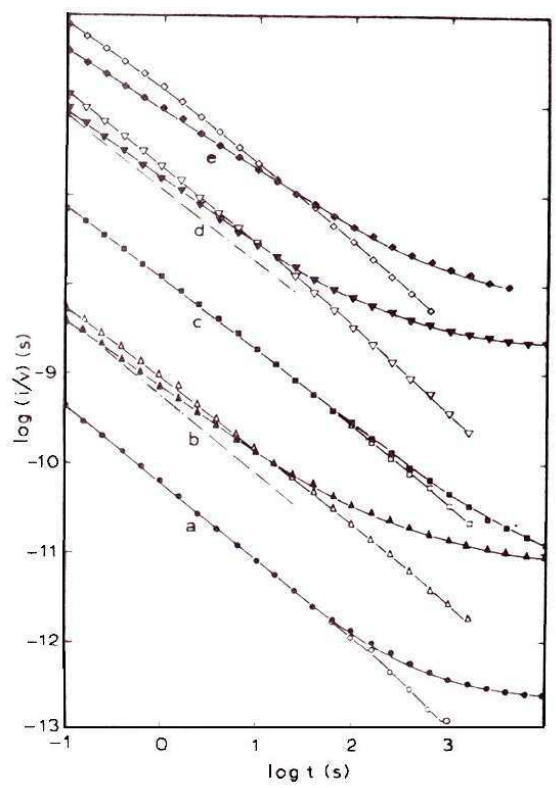

Fig. 3. The logarithmic representation of the charging currents $i_{c}(t)$ and discharging currents $i_{d}(t)$ for Al-Stearic Acid-Al sandwich structures. The temperature and average voltage were as follows [12].

\begin{tabular}{|llllllc|}
\hline Curves & $\mathrm{a}$ & $\mathrm{b}$ & $\mathrm{c}$ & $\mathrm{d}$ & $\mathrm{e}$ & \\
\hline Average voltage & 6.8 & 6.8 & 1.1 & 9.1 & 4.8 & $\times 10^{7} \mathrm{~V} / \mathrm{m}$ \\
\hline Temperature & 50 & 300 & 300 & 300 & 320 & $\mathrm{~K}$ \\
\hline
\end{tabular}

Space charge carriers are free electrons or holes. Charge carriers have a significant role in the determination of the dielectric response of materials since their injection and removal are strongly non-linear [12]. Moreover, the movement of charge carriers in dielectrics is influenced by several phenomena, which becomes especially visible in time domain measurements [12]. As an example, if two materials come into contact (dielectrics or a dielectric and a metal), a transfer of charge will occur between them. The immediate evidence of charge injection is obtained from the build-up potential at the surface [13], [14].

Jonscher [12] stated that injection process is due to interfacial polarisation, which is also known as space charge polarisation. This arises since the charges are accumulated locally as they drift through material [12]. When the atoms or molecules are subjected to a locally distorted electric field a 
large-scale distortion of the voltage takes place [12]. Inhomogeneous medium effects such as charges that pile up in volume or on the surface of the dielectric and imperfections such as cracks usually occur by interfacial polarisation mainly due to the change in conductivity along the boundaries [12].

\section{A. Nonlinearity in transformer PDC measurements}

One of the authors of this paper studied the influence of different physical parameters on PDC measurement [10] and found two main physical quantities that influence the nonlinearity in PDC measurement are the amplitude of the excitation voltage and temperature.

1) Temperature Instability

A major difference in the properties of transformer oil and paper is that the materials are hydrophobic and hydrophilic, respectively. The temperature change will lead to the migration of moisture between oil and paper through diffusion [15]. Accordingly, a dielectric test may not be able to represent the real condition of the insulation except the temperature is stable and moisture equilibrium between oil and paper has reached. Several studies demonstrated that relaxation of currents is very sensitive to temperature [8], [16] which is due to the increased mobility of the charge carries at elevated temperatures [10]. Therefore, temperature instability poses a significant impact on PDC measurement.

\section{2) Excitation Voltage Amplitude}

Fig. 4 shows the influence of charging voltage on measured PDCs on a 100kVA transformer. Two presented measurements were performed at $500 \mathrm{~V}$ and $1000 \mathrm{~V}$. For easier comparison, the currents measured with $500 \mathrm{~V}$ charging voltage were multiplied by 2 [10].The results show that the corresponding measured depolarisation current measured with $1000 \mathrm{~V}$ charging voltage is lower. The higher excitation voltage causes such non-linearity and this may lead to the errors in computing the dielectric response function and the conductivities [10].

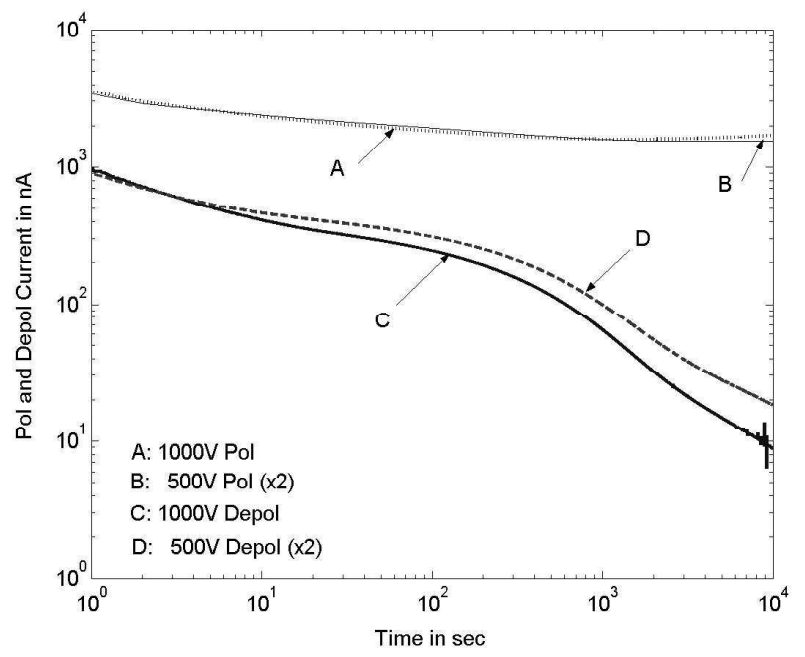

Fig. 4. Relaxation currents at different excitation voltages (scaled to the same level for comparison) [10].

According to Jonscher [12], if the amplitude of the field is raised sufficiently, a complete crossing over of the polarisation and depolarisation currents at short time range may be observed due to the injection of excess space charges into the dielectric during the charging process. Fig. 5 shows an example of a 30MVA transformer with complete crossing over of the depolarisation and polarisation currents at short time range [10].

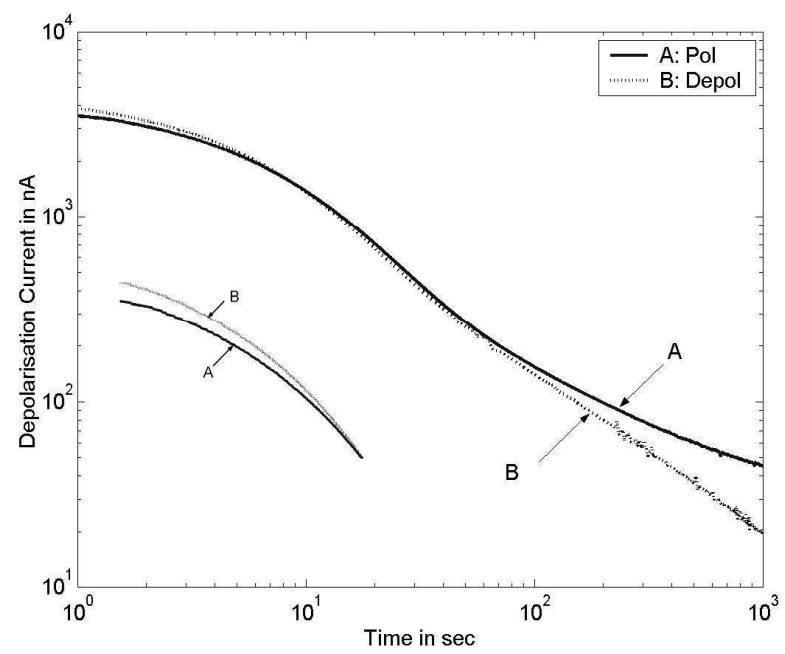

Fig. 5. Crossing over of depolarisation and polarisation currents; Inset shows the initial period in an enlarged scale [10].

\section{ANALySIS OF LABORATORY RESUltS}

This section presents the laboratory results of PDC measurements for investigating the nonlinearity of transformer insulation dielectric response.

\section{A. PDC Test Setup}

PDC measurements were conducted on a $100 \mathrm{kVA}$ oil filled distribution transformer. In the measurements transformer was charged by different voltage levels at room temperature for 5000 seconds. During this period, polarisation current was measured using an electrometer. Then for another 5000 seconds, DC voltage source was disconnected, while the electrometer was measuring the depolarisation current see Fig. 6.

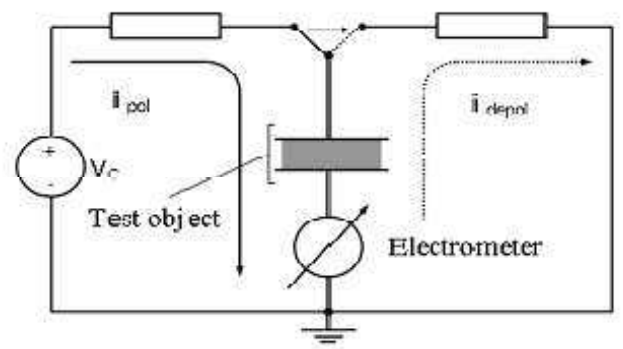

Fig. 6. Simplified internal circuit of PDC measuring device [17]

In order to observe the effect of the step voltage increase on the dielectric response of the transformer insulation, a set of nine tests with different DC voltage levels was performed. Fig. 7 illustrates the measured polarisation currents obtained from these tests.

Theoretically, the initial parts of polarisation currents are more sensitive to the insulation condition of oil however 
currents in long time ranges are dominated by the insulation condition of paper conductivity and moisture content [3].

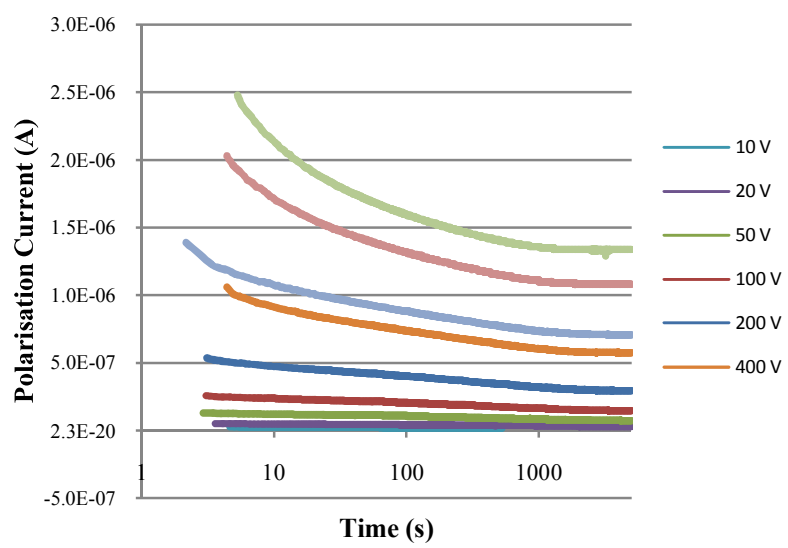

Fig. 7. Measured polarisation currents

\section{B. Analysis of Nonlinearities in Polarisation Currents}

This section investigates the nonlinearity in polarisation currents, which is in response to an increase in the voltage level. The simplest way to identify the existence of such nonlinearity is to plot the actual current and the expected linear current in the same graph. In order to obtain the linear current, it is assumed that the base current was attained at the lowest voltage level $(10 \mathrm{~V})$. Accordingly, the expected linear current at a higher voltage will be a multiplication of the base current. For example, if the applied voltage $(10 \mathrm{~V})$ increases by a factor of two, then the base current will be multiplied by factor two. The measured currents and the expected linear currents in low voltages $(10,40$ and $50 \mathrm{~V})$ were similar. However, when the applied voltage is increased to $100 \mathrm{~V}$ or above, the measured current slowly drifts apart from the corresponding linear one. Fig. 8 shows that the difference between each of the two current curves with the gradually increased voltage. The result implies that the nonlinearity is due to the decrease in the measured current compared to the expected linear current.

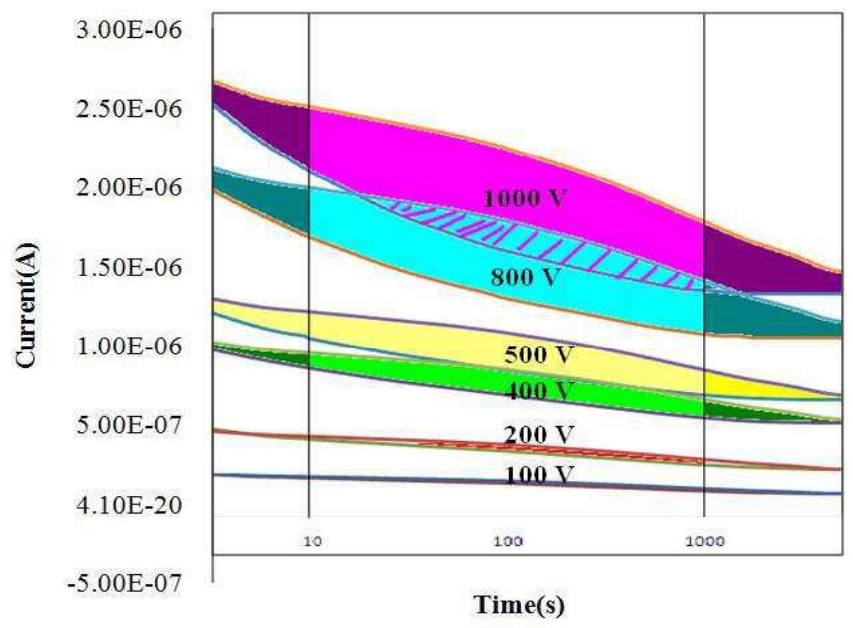

Fig. 8. Actual polarisation current and expected linear current

\section{Computation of Equivalent Model Parameters}

A computer program has been developed by one of the authors of this paper to calculate the RC values of a linear equivalent circuit for dielectric response of transformer insulation system (Fig. 1) [18]. The geometric capacitance $\mathrm{C}_{0}$ in this circuit is equal to measured capacitance at $50 \mathrm{~Hz}$ divided by relative permittivity. After obtaining the above parameters ( $\mathrm{RC}$ and $\mathrm{C}_{0}$ values), the equivalent $\mathrm{RC}$ circuits of the above transformer can be calculated. The corresponding parameters of equivalent $\mathrm{RC}$ circuits for the $500 \mathrm{~V}$ step voltage are as shown in Fig.9.
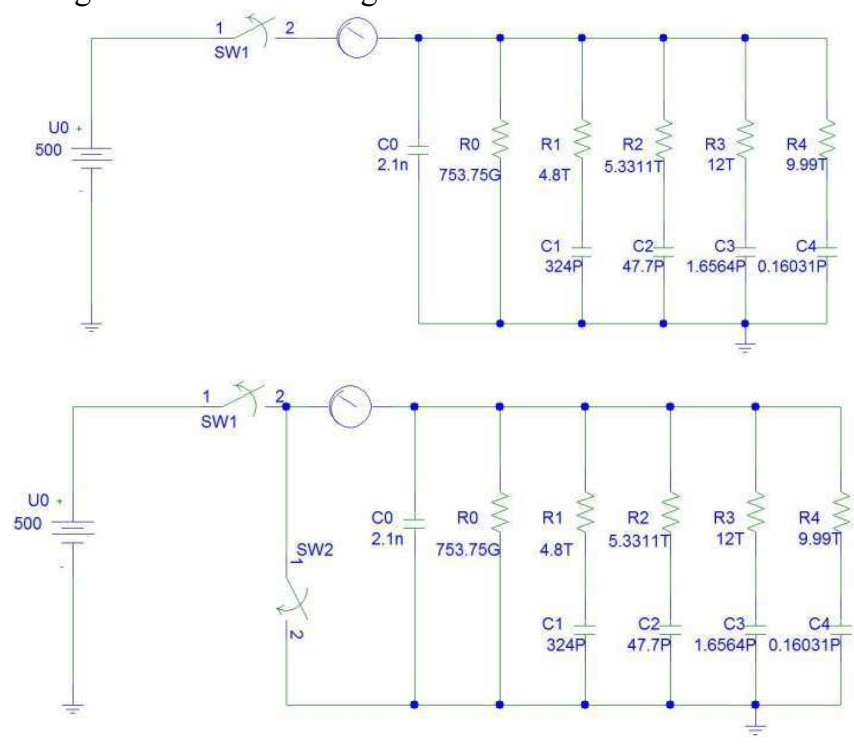

Fig. 9. a) Polarisation equivalent circuit for $500 \mathrm{~V}$ step voltage, b) Depolarisation equivalent circuit for $500 \mathrm{~V}$ step voltage

\section{Proposed Model of Transformer Diagnosis NONLINEARITY}

After confirming the existence of nonlinearity, an algorithm is developed to represent the nonlinear behaviour exhibited in the system. The process began by specifying the model requirements. According to the Fig. 8, the current nonlinearity appeared at a voltage level above $100 \mathrm{~V}$. To evaluate the effect of voltage on the nonlinear phenomenon in transformer insulation dielectric response the polarisation current interval has been divided into three regions: 1-10 second (oil condition), 10-1000 second (interfacial region), and 10005000 second (paper condition) [3]. It is important to note that the most severe nonlinearity occurred at 100 seconds, which is in the oil/paper interfacial region (refer to Fig. 8) [3].

\section{A. Modelling procedure}

To model the polarisation current, the relation between current, time and applied voltage has been derived. This is expressed as:

$$
\begin{aligned}
& i_{p o l}(t)=C_{1} t^{-n} \\
& i_{p o l}(v)=C_{2} v^{m}
\end{aligned}
$$

Where $\mathrm{C}_{1}$ is a constant and $\mathrm{n}$ is the power index of polarisation current with respect to time. Similarly $C_{2}$ is a 
constant and $\mathrm{m}$ is the power index value of polarisation current with respect to voltage. The main difference between the above two equations is in the polarity of their power factor considering $\mathrm{n}$ and $\mathrm{m}$ as positive numbers.

The primary goal for modelling polarisation current is to propose an equation for current as a function of time and voltage. Thus, Equation 7 has been determined by combining Equations 5 and 6.

$$
i_{p o l}(t, v)=\left(v^{m}\right) \times\left(A t^{-n}\right)
$$

In order to assess the accuracy of Equation 7, a MatLab surface fitting tool is used. The value of time and voltage were entered as the inputs and the measured current was entered as the output. Then Equation 7 was entered to fit the surface accordingly. Table 1 shows the obtained errors. It can be seen that Equation 7 exhibits a good fit.

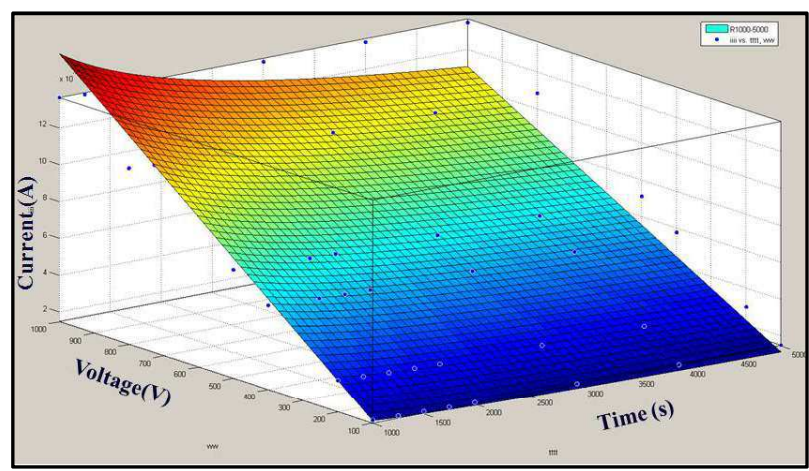

Fig. 9. MatLab surface fitting tool box

TABLE 1

FIT GOODNESS TABLE

\begin{tabular}{|l|l|l|}
\hline Interval & R-Square & RMSE \\
\hline $1-10$ & 0.9953 & $4.97 \mathrm{e}-8$ \\
\hline $10-1000$ & 0.9595 & $1.05 \mathrm{e}-7$ \\
\hline $1000-5000$ & 0.9418 & $1.03 \mathrm{e}-7$ \\
\hline
\end{tabular}

TABLE 2

VARIATION OF A, M AND N IN EQUATION 7

\begin{tabular}{|l|l|l|l|}
\hline & $\mathbf{1 - 1 0}$ & $\mathbf{1 0 - 1 0 0 0}$ & $\mathbf{1 0 0 0 - 5 0 0 0}$ \\
\hline $\mathrm{m}$ & 0.97 & 0.93 & 0.99 \\
\hline $\mathrm{A}$ & $3.7 \mathrm{e}-9$ & $5.07 \mathrm{e}-7$ & $7.9 \mathrm{e}-9$ \\
\hline $\mathrm{n}$ & 0.16 & 0.15 & 0.23 \\
\hline
\end{tabular}

Along with evaluating the accuracy of Equation 7, the calculations have also been done with different parameters as shown in Table 2. In Table 2, the magnitude of $\mathrm{m}$ and $\mathrm{n}$ varies in a range of $0.93-1$ and $0.15-0.23$ respectively. Therefore, the influences of voltage and time on the output polarisation current are limited in small ranges. On the other hand, the comparison of $\mathrm{A}$ in three regions shows that it varies in large range over time. The value of $\mathrm{A}$ can be related to the other parameters in transformer insulation system. Equation 8 has been derived to determine value of $\mathrm{A}$.

$$
A=\frac{C_{0}}{\varepsilon_{0}} \sigma \times B
$$

Where $\mathrm{C}_{0}$ is the geometric capacitance and $\sigma$ is the conductivity of transformer insulation system. In this equation, coefficient $B$ has been assigned as an indication of conductivity change in each region due to the applied high voltage. To verify the accuracy of the above equations, the calculated polarisation current from Equation 7 and 8 has been plotted along with the measured current. As can be seen in Fig. 10, there is not much difference between measured and calculated current which have RMSE value of 4.63 E-8.

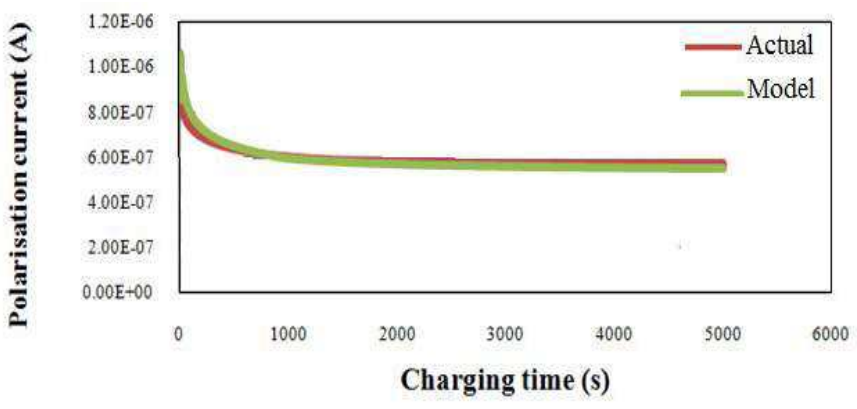

Fig. 10. Original current and calculated current from model

\section{B. Modification in Linear RC Circuit}

Using the available Linear RC circuit, it is not possible to simulate the nonlinear dielectric response characteristics of transformer insulation systems. Therefore, it is necessary to modify current linear model to accommodate the nonlinear properties of transformer insulation dielectric response. As discussed previously, the main reason of dielectric nonlinear phenomenon is the injection process occurs in the interfacial region of two dielectrics material. It was also shown in the experiment section that for the testing transformer, the nonlinearity mainly occurs at 100 seconds, which belongs to oil/paper interfacial region. The electrochemical reactions in the insulation materials could be defined as a source of voltage or current. As a result, a dependent source can be introduced to simulate nonlinear phenomenon.

\section{1) Effects of CCCS}

By considering the injection current phenomenon in the interfacial region, it has been thought that the current controlled current source (CCCS) might be an appropriate component that can be augmented to the linear circuit. However obtained polarisation current in simulated result is higher than expected linear current while non-linearity causes in decreasing of the polarisation current.

\section{2) Effects of CCVS}

The current controlled voltage source (CCVS) is another dependent source which has been examined in this study. Fig 11 shows that a CCVS has been connected in the last two branches of RC circuit which is belong to the dielectric response of interfacial region of oil/paper insulation [3]. In the output figure, the expected linear current is shown in blue colour and actual measured current in red while the green line indicates the obtained current from the modified circuit. 

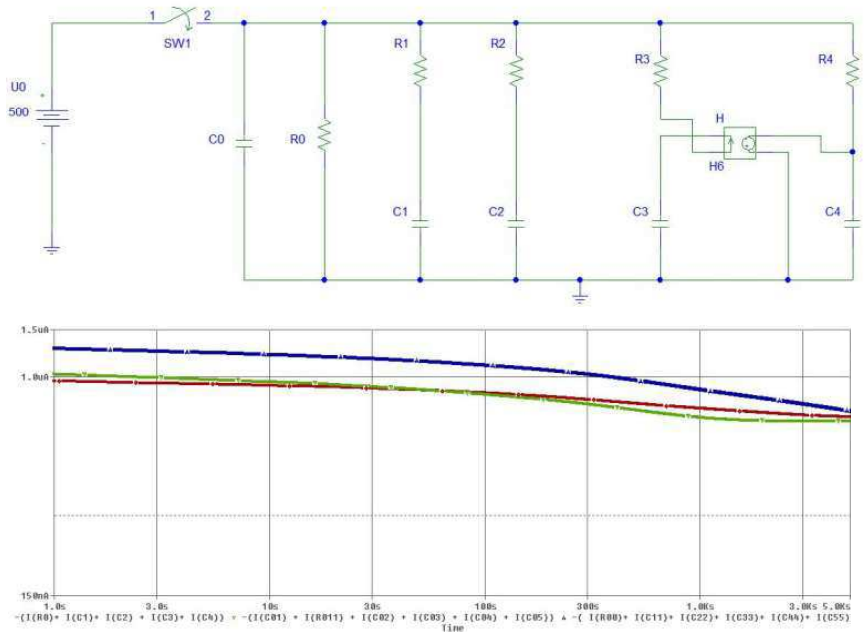

Fig. 11. Addition of CCVS in RC circuit of $500 \mathrm{~V}$ excitation voltage: a) Circuit diagram and b) Simulation result

It can be seen that the output current from the nonlinear circuit (green line) has almost a similar pattern as the actual one (red line). Theoretically, the injection process causes expansion of the barrier between two dielectric materials [19]. As a result the electron space charges as well as the number of electrons captured per time in the barrier will increase [19]. This phenomenon decrease the polarisation current mainly in the barrier of the two dielectrics [19]. Hence, the dependence of current on applied voltage results CCVS appropriate nonlinear component to demonstrate the nonlinear behaviour in the transformer insulation system.

\section{CONCLUSION AND FUTURE WORK}

This paper investigated the nonlinear behaviour of transformer insulation system in dielectric response measurements. Polarisation and depolarisation current (PDC) measurement is performed on a distribution transformer under different voltages to study the nonlinearity of its dielectric response. Based on these measurements a nonlinear transformer insulation model is proposed, in which a current controlled voltage source is augmented to the well-known Debye model to approximate the nonlinear effect.

The findings in this study contribute several proposals for future research:

- This study analysed the impact of high voltage on the nonlinearity of a dielectric response however, high temperature is another factor of nonlinearity. Investigation into the effects of temperature would be beneficial to the understanding of nonlinearity in this context.

- The proposed model was derived by using the measurements from only one transformer. However, conducting measurements on a number of transformers can help to develop a universal model.

- A number of transformers with different age and insulation condition should be explored in depth to examine the effects of insulation aging and conductivity in nonlinearity phenomenon.

\section{REFERENCES}

C. E. Lin, et al., "An expert system for transformer fault diagnosis using dissolved gas analysis," Power Delivery, IEEE Transactions on, vol. 8, pp. 231-238, 1993.

M. D. Judd, et al., "Partial discharge monitoring of power transformers using UHF sensors. Part I: sensors and signal interpretation," Electrical Insulation Magazine, IEEE, vol. 21, pp. 5-14, 2005.

T. K. Saha, et al., "Deriving an equivalent circuit of transformers insulation for understanding the dielectric response measurements," Power Delivery, IEEE Transactions on, vol. 20, pp. 149-157, 2005.

G. M. Urbani and R. S. Brooks, "Using the recovery voltage method to evaluate aging in oil-paper insulation," in Conduction and Breakdown in Solid Dielectrics, 1998. ICSD '98. Proceedings of the 1998 IEEE 6th International Conference on, 1998, pp. 9397.

A. K. a. T. Bedel, "Dielectric diagnosis of water content in transformer insulation systems," Eur. Trans. Electr. Power, vol. 11, pp. pp. 65-68, 2001.

G. Mohamed and E. Németh, "Computer simulation of dielectric processes," presented at the Int. Symp. High Voltage Engineering, Dresden, Germany, 1991.

P. R. S. Jota, et al., "Modeling the polarization spectrum in composite oil/paper insulation systems," Dielectrics and Electrical Insulation, IEEE Transactions on, vol. 6, pp. 145-151, 1999.

T. Leibfried and A. J. Kachler, "Insulation diagnostics on power transformers using the polarisation and depolarisation current (PDC) analysis," in Electrical Insulation, 2002. Conference Record of the 2002 IEEE International Symposium on, 2002, pp. 170-173.

V. Der Houhanessian and W. S. Zaengl, "Application of relaxation current measurements to on-site diagnosis of power transformers," in Electrical Insulation and Dielectric Phenomena, 1997. IEEE 1997 Annual Report., Conference on, 1997, pp. 45-51 vol.1.

T. K. Saha and P. Purkait, "Some precautions for the field users of PDC measurement for transformer insulation condition assessment," in Power Engineering Society General Meeting, 2003, IEEE, 2003, p. 2376 Vol. 4.

$\mathrm{X} . \mathrm{Yu}$ and Birlasekaran, "Study of recovery voltage mechanism [transformer insulation diagnosis]," in Properties and Applications of Dielectric Materials, 2000. Proceedings of the 6th International Conference on, 2000, pp. 935-938 vol.2.

A. K. Jonscher, Dielectric Relaxation in Solids: Chelsea Dielectric Press, 1983.

R. M. H. R. J, "Solid State Physics " Phys.C, vol. 8, pp. 2488$2501,1975$.

C.Rose-Innes, Physics of dielectric Solids, p. 122, 1981.

Y. Sheiretov and M. Zahn, "Dielectrometry measurements of moisture dynamics in oil-impregnated pressboard," Dielectrics and Electrical Insulation, IEEE Transactions on, vol. 2, pp. 329-351, 1995.

U. Gafvert and E. Ildstad, "Modelling return voltage measurements of multi-layer insulation systems," in Properties and Applications of Dielectric Materials, 1994., Proceedings of the 4th International Conference on, 1994, pp. 123-126 vol.1.

A. Setayeshmehr, et al., "Dielectric spectroscopic measurements on transformer oil-paper insulation under controlled laboratory conditions," Dielectrics and Electrical Insulation, IEEE Transactions on, vol. 15, pp. 1100-1111, 2008.

F. Muller, "measurements and simulations of Diagnostic Quantities for Power Transformer Diagnosis," Deploma, The University of Queensland,, 2003.

J. K. a. K.H.Gundlach, "Dependence of Current Decrease opt Magnitude of loading Voltage " Simulation engineering, 1972. 


\section{BIOGRAPHIES}

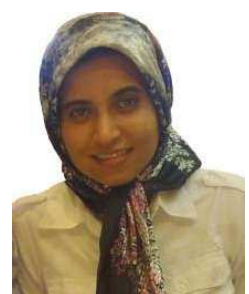

Atefeh Dehghani Ashkezari (SM'10) was born in Iran. She received her B. Eng (electrical and computer engineering) in 2009 from Sohar University, Oman and M.Eng (electrical engineering) in 2010 from the University of Queensland, Australia. She completed her master's thesis on non-linearity in transformer diagnosis. She is currently a research higher student at the University of Queensland. Her research interests include condition monitoring, transformer diagnosis and power system.

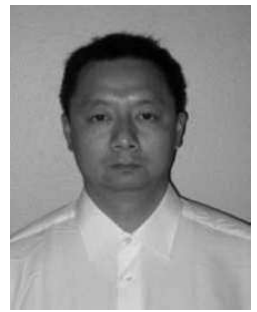

Hui Ma (M'1995) was born in Xi'an, China. He received his B.Eng and M.Eng degrees from Xi'an Jiaotong University, Xi'an, China in 1991 and 1994, M.Eng (research) from Nanyang Technological University, Singapore in 1998, and Ph.D. degree from the University of Adelaide, Adelaide, Australia in 2008. Currently Dr. Ma is postdoctoral research fellow in the School of Information Technology and Electrical Engineering, the University of Queensland, Brisbane, Australia. Prior to joining the University of Queensland, Dr. Ma has many years research and development experience. From 1994 to 1995, he was a researcher in Xi'an Jiaotong University, China. From 1997 to 1999, he worked as a firmware development engineer in CET Technologies Pte. Ltd., Singapore. He was with Singapore Institute of Manufacturing Technology as a research engineer from 1999 to 2003. Dr. Ma's research interests include industrial informatics, condition monitoring and diagnosis, power system, wireless sensor networks, and sensor signal processing.

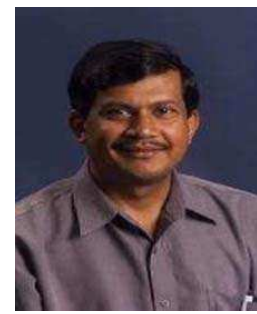

Tapan Kumar Saha (M'93, SM'97) was born in Bangladesh in 1959 and immigrated to Australia in 1989. He received his B. Sc Engineering (electrica and electronic) in 1982 from the Bangladesh University of Engineering \& Technology, Dhaka, Bangladesh, M. Tech (electrical engineering) in1985 from the Indian Institute of Technology, New Delhi, India and PhD in 1994 from the University of Queensland, Brisbane, Australia. Tapan is currently Professor of Electrical Engineering in the School of Information Technology and Electrical Engineering, University of Queensland, Australia. Previously he has had visiting appointments for a semester at both the Royal Institute of Technology (KTH), Stockholm, Sweden and at the University of Newcastle (Australia) $\mathrm{He}$ is a Fellow of the Institution of Engineers, Australia. .His research interests include condition monitoring of electrical plants, power systems and power quality.

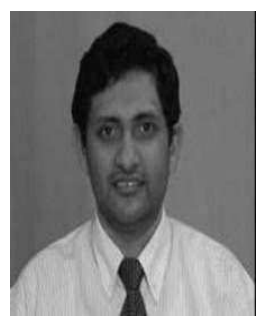

Dr. C.M.B. Ekanayake (M' 00) received his B.Sc.Eng.(Hons) in 1999 from University of Peradeniya. He obtained his Tech. Lic. and PhD from Chalmers University of Technology Sweden in 2003 and 2006 respectively. Currently he is a postdoctoral research fellow in the School of Information Technology and Electrical Engineering, the University of Queensland, Brisbane, Australia. Before joining UQ he was attached to the department of Electrical and Electronic Engineering, University of Peradeniya Sri Lanka as a Senior lecturer. During his PhD studies he was working for a European Union Project called REDIATOOL where he engaged in research related to Diagnostics of Transformer Insulation from dielectric response measurements. Also he has carried out severa dielectric response measurements at ETH, Switzerland as a part of his research work. From 2001, he has been involving on condition monitoring of transformers installed at Ceylon Electricity Board, Sri Lanka. He was the Chair of IEEE Sri Lanka Section in year 2006 and 2007. His research interests are condition monitoring of power apparatus, Alternatives for insulating oil, transient studies on power systems and energy related studies. 